\title{
Tagungsbericht
}

\section{Jahre Ingenieurarbeit. Naturbeherrschung und Weltveränderung}

Technikgeschichtliche Jahrestagung des Vereins Deutscher Ingenieure vom 2. bis 3. März 2006 in Düsseldorf

Das 150jährige Jubiläum des VDI bildete den Rahmen der diesjährigen Technikgeschichtlichen Jahrestagung, die am 2. und 3. März 2006 in Düsseldorf stattfand. In enger Anlehnung an den zu diesem Anlass herausgegebenen Sammelband zur Berufsgeschichte des Ingenieurs ${ }^{1}$ stand die Tagung unter dem Oberthema 6000 Jahre Ingenieurarbeit. Naturbeherrschung und Weltveränderung. In seinen Grußworten gab der Präsident des VDI Eike Lehmann zugleich die Auslobung des „Conrad-Matschoß-Preises“ bekannt, der zukünftig jedes Jahr für herausragende technikhistorische Forschungsarbeiten verliehen werden soll. Walter Kaiser (Aachen), Vorsitzender des Bereichs Technikgeschichte im VDI, stellte in seiner Einführung in das Tagungsthema kursorisch die einzelnen Stationen der Berufsgeschichte des Ingenieurs vor, wie sie die folgenden Referate von der Frühgeschichte in die Gegenwart nachzeichnen sollten.

Unter dem programmatischen Titel Am Anfang war der Ingenieur verlegte Eva Cancik-Kirschbaum (Berlin) den Beginn des Ingenieurberufs ins Zweistromland sogar 10.000 Jahre vor unsere Zeitrechnung. Sie argumentierte, dass mit der neolithischen Revolution, der Sesshaftwerdung des Menschen im ,fruchtbaren Halbmond“, und der damit einhergehenden arbeitsteiligen Organisation von Gesellschaft der von ihr so genannte „Technische Experte" die Bühne der Geschichte betrat. Haus- und Stadtbau und insbesondere die Errichtung monumentaler Kultbauten förderten die Herausbildung einer eigenständigen Berufsgruppe, die sich das benötigte technische Wissen aneignete und praktisch bereitstellte. Anhand der ältesten überlieferten Berufsnamenliste, der Liste „Lu“ (um 3000 v.Chr.), zeigte die Referentin, dass die Technischen Experten in der gesellschaftlichen Hierarchie sehr hoch standen. Diese herausragende Stellung der Technischen Experten spiegelte sich auch in der sumerischen Götterwelt und der Stilisierung des Königs zum Techniker ,par excellence“. So könne der Gott Enki, der eine Art Mittler zwischen Menschen und Göttern war, auch als Technischer Experte begriffen werden, der den Menschen grundlegende technische Fertigkeiten vermittelte. Und die sumerischen Könige wurden in der Dichtung häufig als

1 Walter Kaiser u. Wolfgang König (Hg.), Geschichte des Ingenieurs. Ein Beruf in sechs Jahrtausenden, München, Wien 2006. 
intelligente Konstrukteure und Baumeister verehrt, die sich dadurch besonders auszeichneten, dass sie gestaltend in die Natur eingriffen und so den Wohlstand der Gesellschaft mehrten.

Zeitlich und inhaltlich unmittelbar anschließend präsentierte Ariel M. Bagg (Berlin) in seinem Beitrag zwei altorientalische Könige als Bauherren und Technische Experten. So ließ König Sanherib (704-681 v.Chr.) für den Ausbau Ninives zur Hauptstadt des assyrischen Reiches vier gewaltige Kanalbauten durchführen. Für die Wasserversorgung der Stadt wurde ferner der erste mit Quadersteinen gebaute Aquädukt errichtet. Auch Festungs-, Stadtund Palastbau zeigten Sanherib als großen Projektmanager. Sichtbar wurde das technische Wissen und die erfolgreiche Realisierung von technischen Projekten während des Feldzugs Sanheribs gegen Judäa. Die Belagerung von Schichach und die dabei eingesetzte Militärtechnik sind Zeugnis der technischen Expertise der assyrischen „Ingenieure“. Zugleich bildete Sanheribs Feldzug den Übergang zum zweiten Beispiel: Hiskia, König von Judäa (727-698 v.Chr.). Dieser reagierte nämlich auf die assyrische Bedrohung mit dem Ausbau und der weiteren Befestigung Jerusalems. Zentrale technische Projekte waren wiederum große Bauten für die städtische Wasserversorgung, darunter ein Meisterwerk des Tunnelbaus, der „Siloah-Tunnel“.

Auch Helmuth Schneider (Kassel) knüpfte in seinem Vortrag eng an die beiden vorhergehenden Referenten an. Er präsentierte römische Infrastrukturprojekte als beredten Ausweis des technischen Könnens der antiken Experten. Zur Kontrastierung wählte er als Einstieg die Beschreibung von Paris im 18. Jahrhundert als „trockene“ und stinkende Stadt, die weder die Wasserversorgung noch -entsorgung sicherstellen konnte. Erst vor diesem Hintergrund, so die Argumentation, könne die Leistung einer antiken Gesellschaft, die diese Probleme gelöst hat, angemessen gewürdigt werden. Es folgten zahlreiche Beispiele für Verkehrs- und insbesondere Wasserversorgungsbauten des römischen Reiches. Die erfolgreiche Realisierung technischer Großprojekte wie Brücken, Wasserdruckleitungen und Aquädukte deutete der Referent als Beleg für die technische Qualifikation der römischen Experten. Neben den ex post Begriff des Technischen Experten stellte der Referent die in der Antike gebräuchlichen Bezeichnungen des Architekten und des Mechanikers, die bereits eine erste Ausdifferenzierung des Tätigkeitsfeldes des „Ingenieurs" andeuteten.

Einen großen Bogen schlug Marcus Popplow (Cottbus) mit seinem Beitrag, der danach fragte, welche Rolle die „neuen“ Medien Zeichnung, Modell und Traktat für die Renaissance-Ingenieure gespielt haben. Er argumentierte, dass die Nutzung dieser drei Medien in der Spätantike weitgehend verloren gegangen und erst im 14. Jahrhundert wiederentdeckt worden sei. Verantwortlich für diese Revitalisierung sei eine neue Stufe in der Komplexität technischer Projekte gewesen. Neben verschiedenen Innovationen in den einzelnen Medien - wie z.B. der Zentralperspektive in der Zeichnung - 
hätten sich, so die These des Referenten, in der Renaissance neue Verwendungskontexte dieser Hilfsmittel entwickelt. Die Zeichnung wurde nicht mehr nur für die Planung, Präsentation und Durchführung technischer Projekte eingesetzt, sondern auch zur Dokumentation technischer Anlagen und der Verwaltung großer technischer Systeme. Das Präsentationsmedium Modell erhielt als neue Verwendungszuschreibung das Experiment. Und schließlich diente das Traktat zur Information einer breiteren Öffentlichkeit und präsentierte erstmals ein kohärentes Bild des Ingenieurs als technischen Gelehrten.

Wolfgang König (Berlin) wählte als einziger Referent einen einzelbiografischen Zugang. Er untersuchte in seiner Fallstudie das Projekt der OderRegulierung des Aachener Wasserbauingenieurs Otto Intze (1843-1904). Das „Jahrhunderthochwasser“ der Oder 1897 verdeutlichte den zum großen Teil technikeuphorischen und zukunftsoptimistischen Zeitgenossen, dass die Gefahr von Überschwemmungen eine der verbleibenden Gefahren war, die technisch bislang nicht beherrscht wurden. Dagegen stellte Intze sein Projekt zur Oder-Regulierung durch den Bau von zunächst 20 kleineren Talsperren. Im Verlaufe des Projekts, das schließlich 1900 im schlesischen Hochwasserschutzgesetz seinen Abschluss fand, vereinte Intze Expertise, Exekutive und Legislative in seiner Person und nahm so maßgeblichen Einfluss auf die Politik. Seine Fachkompetenz brachte er in Gutachten ein, durch die Projektleitung nahm er auf die Exekutive und schließlich als Mitglied des preußischen Herrenhauses auch auf die Legislative Einfluss. Vier allgemeine Kernfragen destillierte der Referent aus der Diskussion um den technischen Hochwasserschutz: Erstens nach den Systemgrenzen, zweitens nach der Zweckmäßigkeit, drittens nach der ökonomischen Rechtfertigung und viertens nach der Sicherheit. Wobei Intze alle Fragen zugunsten seines Talsperrenbauprojektes beantwortete. Zwar musste er sich - was die Zweckmäßigkeit betraf - zunächst selbst bekehren, aber in der Frage der Sicherheit befielen ihn keine Zweifel. Er bezeichnete die von ihm geplanten Staudämme schlichtweg als „absolut sicher“. Zusammenfassend kam der Referent zu dem Schluss, dass der insgesamt geringen direkten politischen Partizipation der Ingenieure im Wilhelminischen Kaiserreich einzelne Ingenieure gegenüberstanden, die zumeist dank der persönlichen Protektion durch Wilhelm II. großen Einfluss auf die Gesetzgebung ausüben konnten.

Kees Gispen (Mississippi, USA) stellte in seinem Beitrag die Frage nach Gemeinsamkeiten und Unterschieden des Ingenieurberufs in Großbritannien und den USA im 19. Jahrhundert. Die Gemeinsamkeiten der angelsächsischen Ingenieure lagen - zumindest in der kontinentaleuropäischen Fremdzuschreibung - in ihrer praktisch-empirischen Ausbildung, ihrer mangelnden Geduld mit „unnötiger“ Theorie, der Unterscheidung zwischen Ingenieuren und Technikern und dem besonderen Fokus auf Industrie und Wirtschaft. Der Referent stellte diesen Gemeinsamkeiten eine ganze Reihe von Unterschieden gegenüber. So zeigte er zunächst, dass sich der Ingenieurberuf zeit- 
lich sehr verschieden entwickelt hat, was sich nicht zuletzt aus dem unterschiedlichen zeitlichen Verlauf der Industrialisierung in Großbritannien und den USA ergeben habe. Des Weiteren konstatierte der Referent strukturelle Unterschiede, die u.a. in der Betriebsgröße und den Produktionsformen lagen. Die USA tendierten nach dem Bürgerkrieg zum Großbetrieb mit Massenfertigung, während in Großbritannien weiterhin kleine und mittlere Betriebe mit individueller Fertigung dominierten. Mit der Eröffnung von West Point 1802 - gegründet nach dem Vorbild der École Polytechnique in Paris - kam es zur sehr frühen Herausbildung von technischen Fachschulen in den USA. Seit 1849 wurde in den USA bereits das Diplom eines "Civil Engineers“ verliehen, während man sich in Großbritannien noch in den 1850er Jahren über die theoretische Ausbildung von Ingenieuren lustig machte. Auch wurde das britische Schulwesen nur sehr zögerlich an die Bedürfnisse der Ingenieurausbildung angepasst. Besonders eklatant unterschieden sich die britischen von den amerikanischen Ingenieuren in ihrer sozialen Stellung, die sich auch aus den jeweiligen Karrieremöglichkeiten in den Betrieben ergab. So rekrutierten sich die amerikanischen Ingenieure aus der unteren Mittelschicht und konnten sich über ihre Ausbildung an Spezialschulen und Technischen Hochschulen Hoffnung auf eine Karriere im Management machen. Dagegen besaßen die britischen Ingenieure große Nähe zur Arbeiterschicht und die „höheren“ Ingenieure versuchten, sich über den Erwerb klassisch humanistischer Bildung von ihnen abzusetzen. Abschließend warf der Referent daher die Frage auf, ob es überhaupt den angelsächsischen Ingenieurtypus gab.

Technik, Geschlecht und Kalter Krieg bildeten für Karin Zachmann (München) die Koordinaten des Berufsfeldes der Ingenieure in der DDR. Der Kalte Krieg diente als Bühne für die Aufführung von Technik, die wiederum als Maßstab für die Systemüberlegenheit genutzt wurde. Dabei stand die Ingenieurausbildung in der DDR durchaus in gesamtdeutschen Traditionen: So wurde die Trennung zwischen Technischer Hochschule und Universität aufrechterhalten, die Schulkultur dominierte weiter über die Praxiskultur und auch die alten technischen Kernbereiche - Maschinenbau, Elektrotechnik und Bauingenieurwesen - behielten ihre Stellung bei. Als Grundlinien für die Veränderungen des Ingenieurberufs benannte die Referentin Verstaatlichung, Zentralisierung und partielle Feminisierung. DDR-spezifische Entwicklungen waren die Herausbildung von technischen Spezialschulen, die besondere Förderung des Ingenieur-Fernstudiums und die Einführung einer Frauenquote für das Ingenieurstudium. Abschließend zog die Referentin ein ambivalentes Fazit der ostdeutschen Ingenieurgeschichte. Auf der einen Seite stand demnach der Erfolg bei der Akkumulation technischer Wissensbestände, so gab es Ende der 1980er Jahre in Ostdeutschland genauso viele Ingenieurinnen und Ingenieure wie in Westdeutschland. Auf der anderen Seite standen aber politische Blockaden durch das Herrschaftssystem, die u.a. zu 
zwei weitreichenden Fehlentscheidungen führten: Nämlich dem gescheiterten Aufbau einer eigenen Flugzeugindustrie und dem Einschlagen des schwerindustriellen Entwicklungspfades, der den Strukturwandel der ostdeutschen Wirtschaft nach der Wiedervereinigung massiv erschwerte.

Walter Kaiser (Aachen) fragte in seinem Beitrag nach den Veränderungen der Ingenieurtätigkeit durch den Einsatz von digitaler Rechentechnik, genauer von Computer Aided Design (CAD), Finite Elemente Methode (FEM) und Virtual Reality (VR). Dabei betonte er die Rückgebundenheit der Entwicklung dieser Verfahren an die jeweils verfügbare Computertechnik. So geht CAD wesentlich auf die Arbeiten von Donald Sutherland am Bostoner MIT Anfang der 1960er Jahre zurück, war aber in besonderer Weise auf die Entwicklung von leistungsfähigen und gut handhabbaren Ein- und Ausgabegeräten angewiesen. Daneben war das durch den französischen Mathematiker Pierre Bézier entwickelte - und nach ihm benannte - Verfahren zur Darstellung und Manipulation von beliebigen Krümmungen am Rechner bedeutsam. Erst dadurch konnten Freiformflächen in den computergestützten Konstruktionsprozess integriert werden. Die Methode der finiten Elemente basiert vereinfacht auf der Aufgliederung komplexer Bauteile in einfache Abschnitte - also der Entwicklung eines idealisierten Modells aus endlichen Elementen -, die erst die Berechnung des ganzen Bauteils in Form einer Näherungslösung ermöglichen. Erste Projekte waren die Berechnungen von Staumauern und Kühltürmen, während der eigentliche Durchbruch der FEM in der amerikanischen Luftund Raumfahrt in den 1960er Jahren erfolgte. Die Verschmelzung von CAD und FEM sowie die Einbeziehung dynamischer Kräfte in die FEM ermöglichte die rechnergestützte Simulation des Verhaltens technischer Bauteile und stellte insofern eine neue Art der ingenieurwissenschaftlichen Naturbeherrschung dar. Wobei in der FEM zugleich auch die systeminhärenten Grenzen der Naturbeherrschung begründet liegen, stellt sich doch die Frage, inwieweit die auf Näherungslösungen basierende Simulation an unsere Realität rückgebunden ist. Noch deutlicher als bei den vorgenannten Verfahren wird die Rückkopplung an die Hardware bei der Entwicklung der VR deutlich. Erste dahingehende Versuche setzten bereits mit Morton Heiligs Sensorama im Jahre 1960 ein, aber erst Ende der 1980er Jahre war die Computertechnik soweit, diese Ideen praxistauglich umzusetzen. Die Visualisierung technischer Bauteile und Abläufe bildet heute eine eigene Wirklichkeit der Ingenieurtätigkeit. In der Erzeugung künstlicher technischer Welten kann also der vorläufige Endpunkt der ingenieurtechnischen Weltveränderung gesehen werden.

Der Beitrag von Helmut Winkler (Kassel) verließ abschließend die historische Betrachtungsweise und wandte sich ganz dem Ingenieurberuf in der Gegenwart zu. Ausgehend von der Debatte um die Folgen der Globalisierung und Internationalisierung des Industriestandortes Deutschland fragte der Referent zunächst nach den Motiven für die Tätigkeit von Ingenieuren im Aus- 
land. Dabei zeigte sich, dass neben der Entsendung durch den Arbeitgeber die Möglichkeiten zum Erwerb kultureller und sozialer Kompetenzen eine wichtige Rolle spielen. Mit Blick auf die Folgen der Auslandstätigkeit zog der Referent ein ambivalentes Urteil. Für den einzelnen Ingenieur lohnt sich seine Mobilität nicht. Ein zunächst überraschendes Ergebnis; aber Untersuchungen haben gezeigt, dass Ingenieure, die nicht im Ausland waren, im Durchschnitt mehr verdienen als ihre international erfahrenen Kollegen. Dies ist ein durchaus deutsches Spezifikum, denn in den meisten europäischen Nachbarländern lohnt sich die internationale Tätigkeit auch für die Betroffenen. Auf den Wirtschaftsstandort Deutschland bezogen kam der Referent zu dem Ergebnis, dass die internationale Mobilitätsforschung für den deutschen Arbeitsmarkt keinen dauerhaften Verlust an Arbeitskräften feststellen kann und somit der oft zitierte „Brain-Drain“ nicht feststellbar ist.

Insgesamt bot die Tagung ein sehr facettenreiches Bild des Ingenieurberufs, wobei das größte Manko der zeitlich ausgreifenden Perspektive das Fehlen der Mediävistik war. So blieb das Mittelalter eine „Black Box“, in der das technische Wissen der Spätantike verloren ging und erst in der Renaissance zurück ans Tageslicht kam. Erfreulich ist dagegen die Einbeziehung von Frühgeschichte und Antike in das Programm der Technikgeschichtlichen Jahrestagung, auch wenn der ganz große Blick auf die 6000jährige Berufsgeschichte des Ingenieurs etwas unscharf blieb. Zwar klang an, dass das Verhältnis von Theorie und Praxis die Ingenieure schon früh beschäftigte, trotzdem fehlte ein wenig die analytische Klammer zwischen den altorientalischen „Technischen Experten“ und den „Digitalen Ingenieuren“ des beginnenden 21. Jahrhunderts. Die Einzelbeiträge thematisierten eine Vielzahl unterschiedlicher Aspekte - wie die realisierten Projekte, die praktische Tätigkeit, die soziale Stellung oder die Ausbildung von Ingenieuren -, wo aber im Sinne der „longue durée“ die gleichbleibenden und sich nur zäh wandelnden Strukturen der Ingenieurtätigkeit lagen und wo diese dann doch brüchig wurden, blieb am Ende der Tagung weitgehend offen.

Anschrift des Verfassers: Stefan Krebs, RWTH Aachen, Lehrstuhl für Geschichte der Technik, Kopernikusstraße 16, D-52074 Aachen, Email: krebs@histech.rwth-aachen.de. 